\title{
Downregulation of feline sarcoma-related protein inhibits cell migration, invasion and epithelial-mesenchymal transition via the ERK/AP-1 pathway in bladder urothelial cell carcinoma
}

\author{
XUDONG HU ${ }^{1}$, ZHIQIANG ZHANG ${ }^{2}$, ZHAOFENG LIANG $^{3}$, DONGDONG XIE ${ }^{2}$, \\ TAO ZHANG ${ }^{2}$, DEXIN YU ${ }^{2}$ and CAIYUN ZHONG ${ }^{3}$ \\ ${ }^{1}$ Department of Urology, Anqing First People's Hospital, Anqing, Anhui 246000; ${ }^{2}$ Department of Urology, \\ The Second Hospital of Anhui Medical University, Hefei, Anhui 230032; \\ ${ }^{3}$ Department of Nutrition and Food Safety, School of Public Health, Nanjing Medical University, \\ Nanjing, Jiangsu 211166, P.R. China
}

Received April 23, 2015; Accepted September 13, 2016

DOI: $10.3892 / 01.2016 .5459$

\begin{abstract}
Feline sarcoma-related protein (Fer) is a nuclear and cytoplasmic non-receptor protein tyrosine kinase and Fer overexpression is associated with various biological processes. However, the clinicopathological characteristics and molecular mechanisms of Fer expression in bladder urothelial cell carcinoma (UCC) have yet to be elucidated. The present study demonstrated that Fer was significantly upregulated in bladder UCC tissues and cell lines. A clinicopathological analysis suggested that Fer expression was significantly associated with tumor stage, histological grade and lymph node status, and Fer expression was a prognostic factor for overall survival in a multivariate analysis. Furthermore, small interfering RNA (siRNA) was used to silence the expression of the Fer gene in human bladder UCC T24 cells, and was shown to significantly reduce the migration and invasion of the cells. It was also observed that Fer-siRNA caused the T24 cells to acquire an epithelial cobblestone phenotype, and was able to reverse the epithelial-mesenchymal transition of the cells. Subsequently, Fer-knockdown was shown to deactivate the extracellular signal-regulated kinase/activator protein-1 signaling pathway in T24 cells. These results indicated, for the first time, that Fer has a critical role in bladder
\end{abstract}

Correspondence to: Dr Dexin Yu, Department of Urology, The Second Hospital of Anhui Medical University, 678 Furong Road, Hefei, Anhui 230032, P.R. China

E-mail: yudx_urology@126.com

Dr Caiyun Zhong, Department of Nutrition and Food Safety, School of Public Health, Nanjing Medical University, 818 Eastern Tianyuan Road, Nanjing, Jiangsu 211166, P.R. China

E-mail: cyzhong@njmu.edu.cn

Key words: feline sarcoma-related protein, bladder urothelial cell carcinoma, epithelial-mesenchymal transition, small interfering RNA
UCC progression and may be a potential therapeutic target for bladder UCC metastasis.

\section{Introduction}

Bladder cancer is the most common urological malignancy among human urothelial cell carcinomas (UCCs) and accounts for $\sim 90 \%$ of all bladder cancers (1). The prognosis of patients with non-invasive bladder cancer is generally favorable, whereas patients with invasive bladder cancer typically show postoperative distant metastasis or local recurrence following a radical cystectomy (2). Feline sarcoma-related protein (Fer) is a 94-kDa non-receptor protein tyrosine kinase, which was shown to reside in the cytoplasm and nucleus of mammalian cells (3). Previous studies have reported Fer activation or upregulation in numerous cancers, including renal (4), hepatic (5), prostate (6), and triple negative breast cancer (7). Furthermore, it has been reported that Fer is able to modulate cell migration and invasion in numerous cell types (8). However, the biological role of Fer in bladder UCC has yet to be defined, and the molecular mechanisms underlying Fer-mediated cell migration and invasion remain unclear.

Metastasis is commonly associated with the progression of malignancy, and the invasive nature of tumor cells is a major prerequisite to cancer metastasis (9). Cell migration has a critical role in cancer cell invasion and metastasis, and is initiated via activation of the epithelial-mesenchymal transition (EMT) in tumor cells. Molecular alterations in the epithelial marker, E-cadherin, and the mesenchymal markers, $\beta$-catenin, $\mathrm{N}$-cadherin and vimentin, may result in dysfunctional cell-cell adhesion and loss of cell-cell junctions, which are associated with cell phenotype transformation (10). Multitudinous transcription factors, including the Snail-family members, snail family transcriptional repressor 1 (Snail), snail family transcriptional repressor 2 (Slug) and twist family bHLH transcription factor 1 (Twist1), induce the EMT by repressing E-cadherin expression $(11,12)$. The EMT also involves a series of complex changes in numerous signaling pathways, including 
the Wnt, Notch and mitogen-activated protein kinase (MAPK) signaling pathways (13).

The MAPK signaling pathway not only promotes cell differentiation, proliferation and survival, but also mediates oncogenesis (14). In a previous study, the MAPK signaling pathway regulated the expression of the activator protein 1 (AP-1) transcription factor, which regulates the expression of members of the proto-oncogene Jun protein (c-Jun, JunB and JunD) and Fos protein (c-Fos, FosB, Fra-1 and Fra-2) families (15). Extracellular-signal-regulated kinases (ERKs) are key components of the MAPK signaling pathway and abnormal activation of the ERK cascade is associated with metastasis in numerous human cancers (16).

In the present study, Fer expression in bladder UCC tissues and cell lines was assessed, and its prognostic value for survival in patients with bladder UCC was evaluated. In addition, the regulatory effect of Fer on T24 cell migration and invasion, and the EMT process, was investigated and was shown to be mediated via the ERK/AP-1 signaling pathway.

\section{Materials and methods}

Tissue samples. This study was approved by the Second Affiliated Hospital of Anhui Medical University (Hefei, China). Tumor samples from resected specimens were collected from two cohorts of patients with primary bladder UCC that underwent transurethral resection, partial cystectomy or radical cystectomy at the Second Affiliated Hospital of Anhui Medical University between December 2008 and October 2013. Cohort A consisted of 12 patients (10 females, 2 males; median age, 55 years; age range, 45-78 years), from whom fresh tumor samples and adjacent histologically normal bladder tissues were obtained for analysis of Fer mRNA and protein expression. Cohort B comprised 78 patients (68 females, 10 males; median age, 65 years; age range, $45-78$ years), whose paraffin-embedded specimens were used for immunohistochemical analysis. A further 20 paraffin-embedded normal bladder mucosal samples adjacent to the neoplastic bladder tissue were obtained from the same UCC patients, and were used as a control. None of the patients had received preoperative treatment. The tumors were stratified as non-muscle invasive bladder cancer or muscle invasive bladder cancer, according to the 2002 Union for International Cancer Control TNM classification of tumor stage (17). The tumor grade was assigned using the 2004 World Health Organization/International Society of Urological Pathology classification system (18). This study was conducted according to the guidelines in the Declaration of Helsinki. Written informed consent was obtained from all participants.

Cell line culture and maintenance. Bladder cancer cell lines, T24, 5637 and BIU-87, and an immortalized normal human urothelial cell line, SV-HUC-1, were purchased from Type Culture Collection of the Chinese Academy of Sciences (Shanghai, China). T24, 5637 and BIU-87 cell lines were cultured in RPMI-1640 medium (Gibco; Thermo Fisher Scientific, Inc., Waltham, MA, USA) containing 10\% fetal bovine serum (FBS; Hyclone; GE Healthcare Life Sciences, Logan, UT, USA) at $37^{\circ} \mathrm{C}$ in $5 \% \mathrm{CO}_{2}$ and at $95 \%$ humidity. SV-HUC-1 cells were cultured in $\mathrm{F} 12 \mathrm{k}$ medium (Wisent, Inc., St. Bruno, QC, Canada) containing
Table I. Oligonucleotide primer sequences used in RT-qPCR.

\begin{tabular}{ll}
\hline Gene & \multicolumn{1}{c}{ Sequence } \\
\hline Fer & F: 5'-TTCGAGGGCACTGGGTTTTC-3' \\
& R: 5'-TTCCCTTGCCCAGTAATTCTCC-3' \\
MMP-2 & F: 5'-TACAGGATCATTGGCTACACACC-3' \\
& R: 5'-GGTCACATCGCTCCAGACT-3' \\
MMP-9 & F: 5'-TGTACCGCTATGGTTACACTCG-3' \\
& R: 5'-RGGCAGGGACAGTTGCTTCT-3' \\
N-cadherin & F: 5'-TGCCAGTCACTTGCTAACAAAAG-3' \\
& R: 5'-GTGTGCGCTGGGAGAATAAAG-3' \\
3-catenin & F: 5'-AAAGCGGCTGTTAGTCACTGG-3' \\
& R: 5'-CGAGTCATTGCATACTGTCCAT-3' \\
E-cadherin & F: 5'-GACCGAGAGAGTTCCCTACG-3' \\
& R: 5'-TCAGGCACCTGACCCTTGTA-3' \\
Vimentin & F: 5'-ATGACCGCTTCGCCAACTAC-3' \\
& R: 5'-CGGGCTTTGTCGTTGGTTAG-3' \\
Slug & F: 5'-ATACCACA ACCAGAGATCCTCA-3' \\
& R: 5'-GACTCACTCGCCCCAAAGATG-3' \\
Snail & F: 5'-TGAGGCCAAGGATCTCCAGG-3' \\
Twist1 & R: 5'-GGGCAGGTATGGAGAGGAAG-3' \\
& F: 5'-GGGAGTCCGCAGTCTTACGA-3' \\
GAPDH & F: 5'-AGACCGAGAAGGCGTAGCTG-3' \\
& R: 5'-AAGGC-CATGCCAGTGAGCTTC-3' \\
\hline &
\end{tabular}

RT-qPCR, reverse transcription-quantitative polymerase chain reaction; Fer, feline sarcoma-related protein; MMP, matrix metalloproteinase; Slug, snail family transcriptional repressor 2; Snail, snail family transcriptional repressor 1; Twist1, twist family bHLH transcription factor $1 ; \mathrm{F}$, forward; $\mathrm{R}$, reverse.

$10 \% \mathrm{FBS}, 100$ units/ml penicillin and $100 \mu \mathrm{g} / \mathrm{ml}$ streptomycin in an atmosphere containing $5 \% \mathrm{CO}_{2}$ at $37^{\circ} \mathrm{C}$. The activator, epidermal growth factor (EGF; $0.1 \mathrm{ng} / \mathrm{ml}$ ), was obtained from Sigma-Aldrich (Merck Millipore, Darmstadt, Germany). At $70 \%$ confluence, T24 cells transfected with Fer-siRNA were serum-starved overnight, followed by incubation with EGF at $37^{\circ} \mathrm{C}$ for $48 \mathrm{~h}$.

Reverse transcription-quantitative polymerase chain reaction $(R T-q P C R)$. Total RNA was isolated from the cells using RNAiso Plus (Takara Bio, Inc., Otsu, Japan), according to the manufacturer's protocol. Total RNA $(5 \mu \mathrm{g})$ was reverse transcribed into cDNA using the M-MLV First-Strand Synthesis system (Promega Corporation, Madison, WI, USA). cDNA was analyzed in triplicate using the MJ Real-Time PCR system (Bio-Rad Laboratories, Inc., Hercules, CA, USA). The primers used are shown in Table I. qPCR was performed using the Power SYBR Green Master Mix (Takara Bio, Inc.) and the ABI 7300 Real-Time PCR detection system (Applied Biosystems; Thermo Fisher Scientific, Inc.). PCR conditions were as follows: Initial denaturation step at $95^{\circ} \mathrm{C}$ for $15 \mathrm{sec}$, followed by 40 cycles of amplification and quantification at $95^{\circ} \mathrm{C}$ for $10 \mathrm{sec}, 60^{\circ} \mathrm{C}$ for 
Table II. Relationship between Fer protein expression and various clinicopathological parameters in 78 bladder UCC tissues.

\begin{tabular}{|c|c|c|c|c|}
\hline \multirow[b]{2}{*}{ Variable } & \multirow[b]{2}{*}{$\mathrm{n}$} & \multicolumn{2}{|c|}{ Fer expression } & \multirow[b]{2}{*}{ P-value } \\
\hline & & Negative & Positive & \\
\hline Age, years (median 65) & & & & 0.459 \\
\hline$<65$ & 38 & 13 & 25 & \\
\hline$\geq 65$ & 40 & 10 & 30 & \\
\hline Gender & & & & 0.246 \\
\hline Male & 68 & 19 & 49 & \\
\hline Female & 10 & 4 & 6 & \\
\hline Tumor stage & & & & 0.042 \\
\hline $\mathrm{Ta}, \mathrm{T} 1$ & 46 & 18 & 28 & \\
\hline $\mathrm{T} 2, \mathrm{~T} 3, \mathrm{~T} 4$ & 32 & 5 & 27 & \\
\hline Histological grade & & & & 0.023 \\
\hline G1 & 19 & 10 & 9 & \\
\hline G2 & 28 & 8 & 20 & \\
\hline G3 & 31 & 5 & 26 & \\
\hline Tumor multiplicity & & & & 0.803 \\
\hline Unifocal & 52 & 16 & 36 & \\
\hline Multifocal & 26 & 7 & 19 & \\
\hline Lymph node status & & & & 0.014 \\
\hline No & 56 & 21 & 35 & \\
\hline $\mathrm{N} 1, \mathrm{~N} 2$ & 22 & 2 & 20 & \\
\hline
\end{tabular}

Fer, feline sarcoma-related protein; UCC, urothelial cell carcinoma.

$30 \mathrm{sec}$ and $72^{\circ} \mathrm{C}$ for $30 \mathrm{sec}$. Fold changes in expression of each gene were calculated using the comparative quantification method (19).

Western blot assay. Cell lysate was prepared by extracting proteins using RIPA buffer (Fermentas Inc., Burlington, ON, Canada) supplemented with $1 \%$ protease inhibitors (Sigma-Aldrich; Merck Millipore). Protein concentrations were measured using the bicinchoninic acid protein assay (Pierce; Thermo Fisher Scientific, Inc.), after which proteins were diluted to equal concentrations, boiled for $5 \mathrm{~min}$ and separated by $7.5-10 \%$ SDS-PAGE, followed by transblotting onto an Immun-Blot ${ }^{\circledR}$ polyvinylidene fluoride membrane (Bio-Rad Laboratories, Inc.). The membranes were blocked with 5\% non-fat milk in TBST and subsequently probed with primary antibodies overnight at $4^{\circ} \mathrm{C}$. The primary antibodies included: Anti-Fer (cat. no. ab52479; 1:500; Abcam, Cambridge, UK), anti-matrix metalloproteinase (MMP)-2 (cat. no. 4022), anti-MMP-9 (cat. no. 3852S), anti-E-cadherin (cat. no. 4065), anti-vimentin (cat. no. 3295S), anti-N-cadherin (cat. no. 4061S), anti- $\beta$-catenin (cat. no. 9562), anti-Slug (cat. no. 9585P), anti-Snail (cat. no. 3879S), anti-Twist1 (cat. no. 4119S), anti-phospho-ERK1/2 (cat. no. 9911S), anti-p-c-jun (cat. no. 8221S) and anti-p-c-fos (cat. no. 5348S) (all 1:500; Cell Signaling Technology, Inc., Danvers, MA, USA). Rabbit anti-GAPDH polyclonal antibody (cat. no. sc-25778; 1:500; Santa Cruz Biotechnology Inc., Santa Cruz, CA, USA) was used as an internal control. Primary antibodies were detected by incubating the membranes with a horseradish peroxidase (HRP)-conjugated secondary antibody (cat. no. 7071; 1:1,000; Cell Signaling Technology, Inc.) for $1 \mathrm{~h}$ at room temperature. The blots were subsequently developed using an enhanced chemiluminescence detection kit (GE Healthcare Life Sciences) and exposure to film.

Immunohistochemical analysis.Immunohistochemical staining was performed using a Dako Envision System (Dako North America, Inc., Carpinteria, CA, USA), according to the manufacturer's protocol. Briefly, 4- $\mu \mathrm{m}$ paraffin-embedded tissue sections were heated for $1 \mathrm{~h}$ at $65^{\circ} \mathrm{C}$, deparaffinized with xylene and rehydrated using a graded series of ethanol/distilled water. Subsequently, the tissue sections were submerged in EDTA buffer ( $\mathrm{pH} 8.0$ ), heated in a microwave for antigen retrieval, treated with $0.3 \% \mathrm{H}_{2} \mathrm{O}_{2}$ for 15 min to block endogenous peroxidase activity and incubated overnight with a rabbit anti-Fer monoclonal antibody $\left(1: 50 ;\right.$ Abcam) at $4^{\circ} \mathrm{C}$. Next, the tissue sections were washed, incubated with HRP-conjugated antibody at $4^{\circ} \mathrm{C}$ for $30 \mathrm{~min}$ and visualized with diaminobenzidine.

RNA interference (RNAi). siRNAs targeting Fer and negative control siRNA were purchased from Shanghai GenePharma Co., Ltd. (Shanghai, China). The sequences of the three siRNAs targeting Fer were as follows: siRNA1, 5'-AAAGAA ATTTATGGCCCTGAG-3'; siRNA2, 5'-CAGATAGATCCT AGTACAGAA-3'; and siRNA3, 5'-AACTACGGTTGCTGG AGACAG-3'). The sequence of the negative control was as 
A

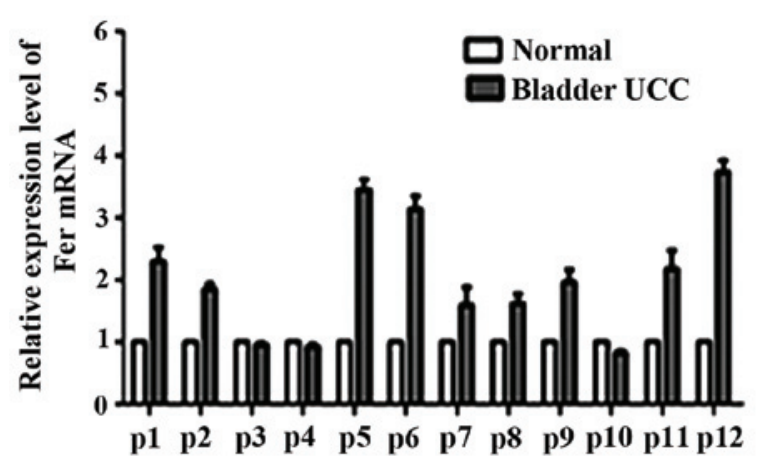

C

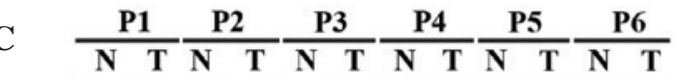

Fer

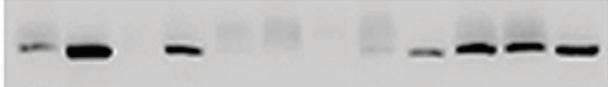

GAPDH

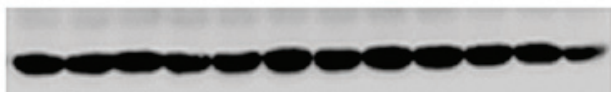

D
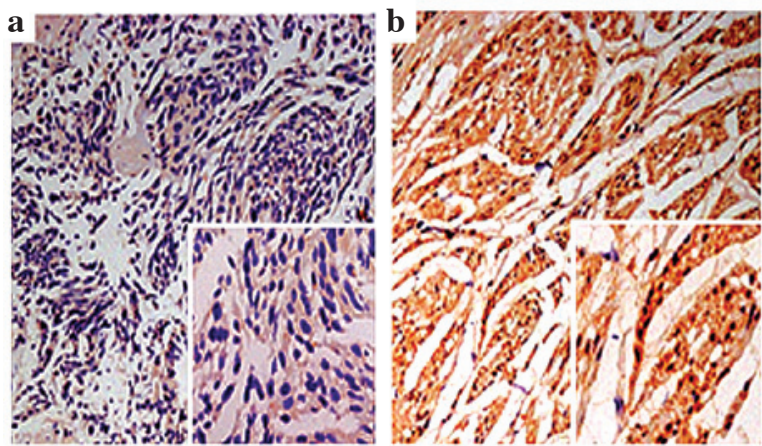
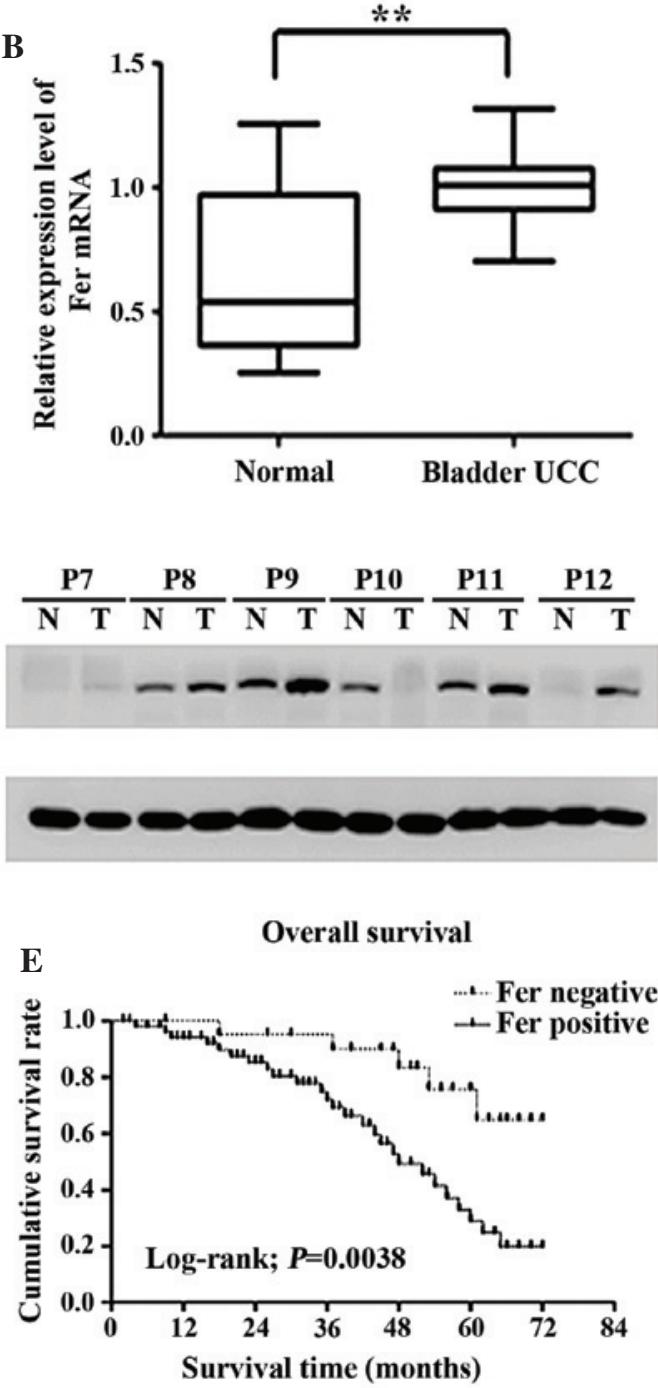

Figure 1. Fer was overexpressed in bladder UCC tissues. (A and B) The mRNA expression levels of Fer were upregulated in bladder UCC tissues compared with their paired adjacent normal bladder tissues, as demonstrated using reverse transcription-quantitative polymerase chain reaction. Data are presented as the mean \pm standard deviation ${ }^{* *} \mathrm{P}<0.01$. (C) Fer protein expression was upregulated in the bladder UCC tissues compared with their paired adjacent normal bladder tissues, as demonstrated using western blotting. GAPDH was used as an internal control. (D) Immunohistochemistry detection of Fer protein expression in normal bladder tissues and bladder UCC tissues: (a) Normal bladder tissues with negative Fer expression; (b) bladder UCC tissues with positive Fer expression (magnification, x200; framed in white, x400). (E) Overall survival analysis using the Kaplan-Meier method revealed that patients with positive Fer expression had markedly lower overall survival rates compared with patients with negative Fer expression. $\mathrm{N}$, adjacent normal bladder tissues; T, bladder UCC tissues; Fer, feline sarcoma-related protein; UCC, urothelial cell carcinoma.

follows: 5'-UUCUCCGAACGUGUCACGU-3'. The sequences of the siRNAs were designed using an RNAi algorithm (https://rnaidesigner.thermofisher.com/rnaiexpress/sort.do). For siRNA transfection, the cells were seeded onto 6-well plates and, after reaching 40-50\% confluency, were transfected with negative control or one of the Fer-specific siRNAs using Lipofectamine 2000 (Invitrogen; Thermo Fisher Scientific, Inc.). Cells were harvested for RNA extraction after $48 \mathrm{~h}$ and protein extraction after $72 \mathrm{~h}$ of transfection.

Cell wound healing assay. T24 cells in 6-well plates were transfected with control or Fer-siRNA. Upon reaching 90-95\% confluence after $24 \mathrm{~h}$, wounds were generated by scratching the surface of the plates with a $0-20 \mu 1$ pipette tip. Wound closure was monitored at various time points $(0,12$ and $24 \mathrm{~h})$ by observation under an inverted microscope, and the degree of cell migration was quantified by the ratio of gap distance at $24 \mathrm{~h}$ to that at $0 \mathrm{~h}$. The experiment was performed in triplicate.

Matrigel invasion assay. Cell invasion assays were performed using 24-well Transwell chambers with a pore size of $8 \mu \mathrm{m}$ (Costar; Corning, Inc., New York, NY, USA). The inserts were coated with $100 \mu \mathrm{l}$ Matrigel (dilution, 1:8; BD Biosciences, Franklin Lakes, NJ, USA). T24 cells were trypsinized following transfection with control or Fer-siRNA for $48 \mathrm{~h}$ and transferred to the upper Matrigel chamber in $100 \mu \mathrm{l}$ serum-free medium for $24 \mathrm{~h}$. The lower chamber was filled with medium containing $10 \%$ FBS as a chemoattractant. Following incubation, the non-invaded cells on the upper membrane were removed using a cotton tip, and the invaded cells on the bottom membrane were evaluated by light microscopy. The cells were stained with crystal violet and the optical density (OD) of the crystal violet 
A

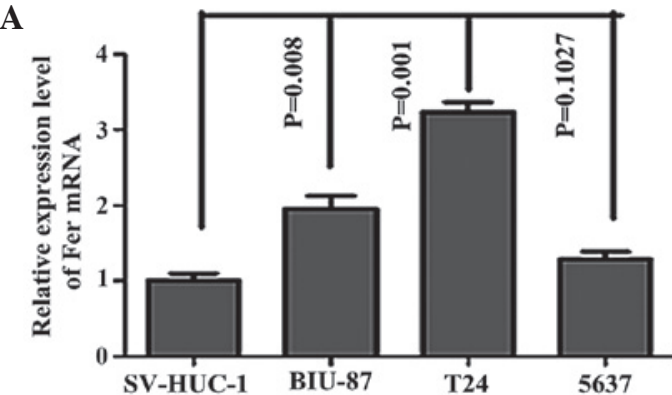

B

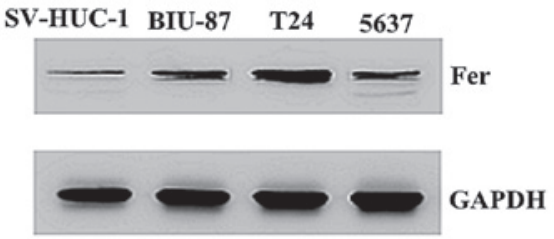

C

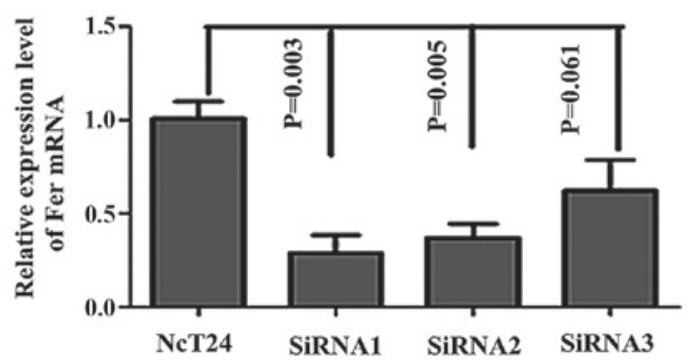

D
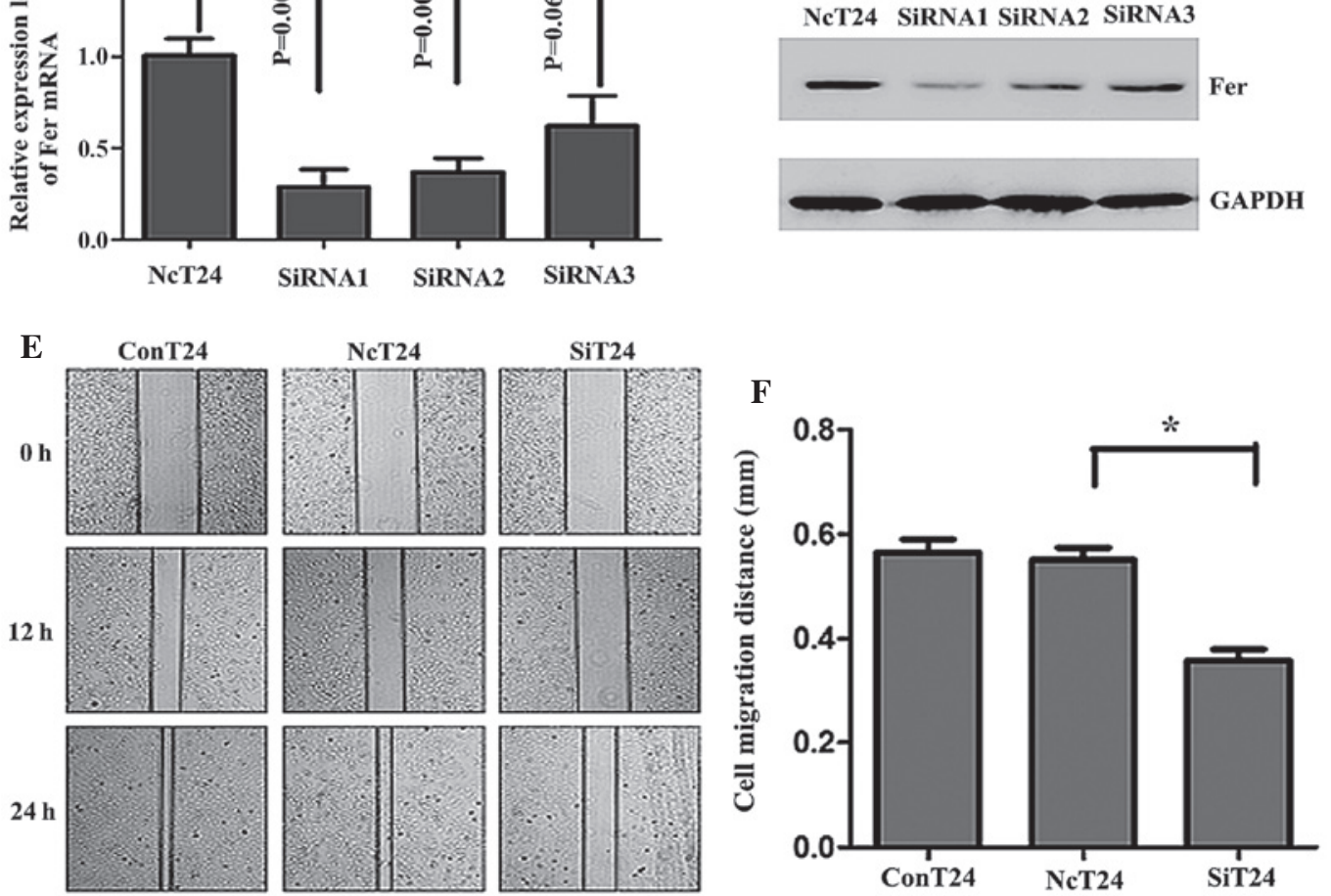

Figure 2. mRNA and protein expression of Fer was upregulated in human bladder cancer cell lines and Fer knockdown using Fer-specific siRNA inhibited the migration of T24 cells. The (A) mRNA and (B) protein expression of Fer in human bladder cancer cell lines and an immortalized normal urothelial cell line, SV-HUC-1, were detected by RT-qPCR and western blotting, respectively. GAPDH was used as an internal control. Data are presented as the mean \pm standard deviation. The transfection efficiency following transfection of T24 cells with Fer-specific siRNAs (siRNA1, siRNA2 and siRNA3) or normal control siRNA for 48 and $72 \mathrm{~h}$ was assessed by (C) RT-qPCR and (D) western blotting. (E) Wound healing assays were conducted to assess the capacity of cell migration in the three groups of cells at 0,12 and $24 \mathrm{~h}$. (F) The relative migration distances were calculated. ${ }^{*} \mathrm{P}<0.05$. The experiments were repeated in triplicate. Fer, feline sarcoma-related protein; siRNA, small interfering RNA; RT-qPCR, reverse transcription-quantitative polymerase chain reaction; NcT24, T24 cells transfected with normal control siRNA; SiT24, T24 cells transfected with Fer-specific siRNAs; ConT24, untreated T24 cells.

solution removed from the cells using $300 \mu$ l glacial acetic acid (33\%) was measured at $570 \mathrm{~nm}$. All experiments were performed in triplicate.

Statistical analysis. Statistical analyses were performed using SPSS software, version 17.0 (SPSS Inc., Chicago, IL, USA). A paired-samples t-test was used to compare the mRNA and protein expression of Fer in the UCC tissues with that in the paired adjacent normal tissue samples. The relationship between Fer protein expression and clinicopathological features was analyzed using $\chi^{2}$ tests. Overall survival curves were constructed using the Kaplan-Meier method and were analyzed using the log-rank test. Paired t-tests and Student's t-tests were used to analyze the findings of the in vitro cell assay. $\mathrm{P}<0.05$ was considered to indicate a statistically significant difference.

\section{Results}

Fer is significantly upregulated in bladder UCC tissues and is correlated with clinicopathological parameters. To investigate the role of Fer in bladder UCC development, the mRNA and protein expression of Fer in 12 bladder UCC tissue samples and adjacent normal bladder tissues were detected by RT-qPCR and western blotting, respectively. As shown in Fig. 1A and B, the relative mRNA expression level of Fer in bladder UCC tissues was significantly higher than that in adjacent normal bladder tissues $(\mathrm{P}<0.01)$, which was consistent with the results of the western blot (Fig. 1C). Immunohistochemical analysis was performed to further analyze the expression of Fer in 78 bladder UCC tissues, as compared with 20 paired adjacent normal tissues. As shown in Fig. 1D, Fer staining was negligible in the normal bladder tissues. Conversely, Fer was positively expressed 
A

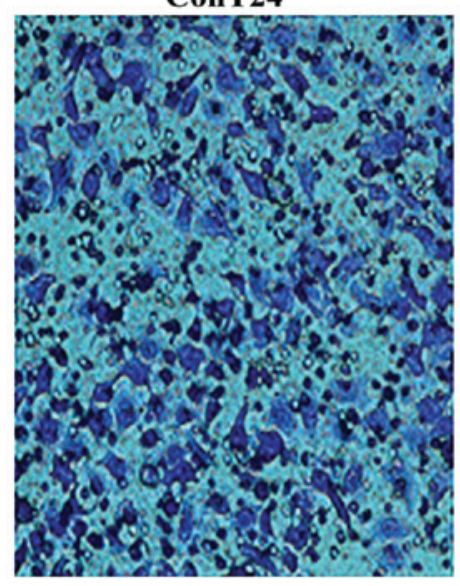

NcT24

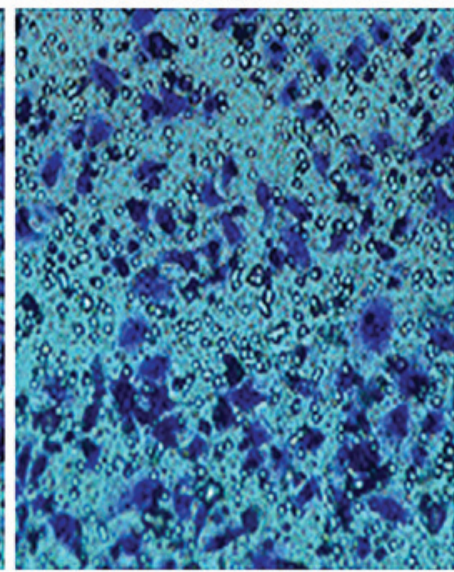

C
B

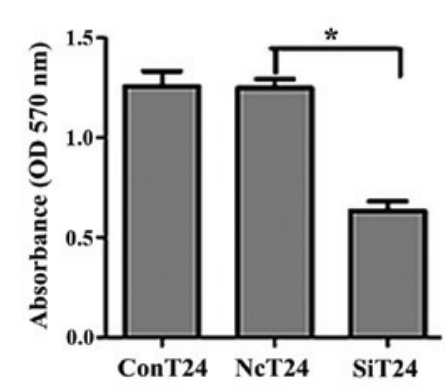

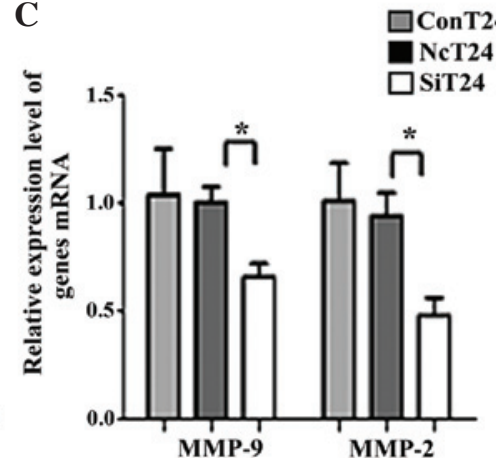

SiT24

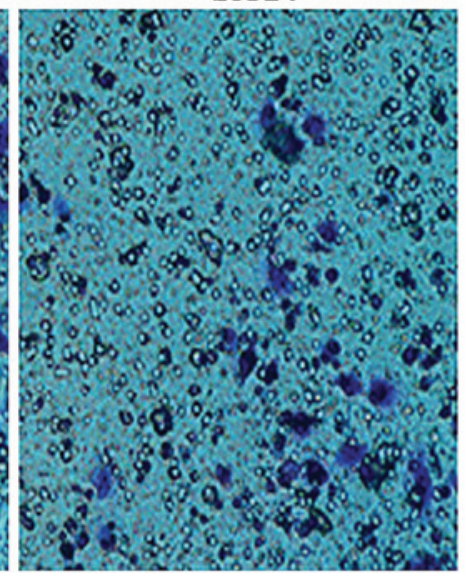

D
ConT24 NeT24 SiT24

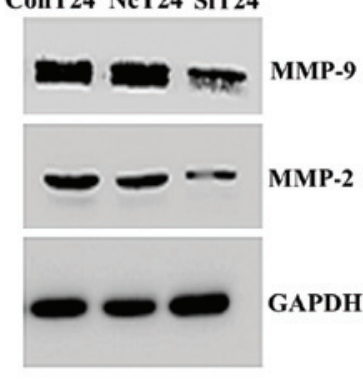

Figure 3. Knockdown of Fer inhibited cell invasion and suppressed MMP family protein expression in T24 cells. (A and B) Matrigel invasion assays demonstrated that cells transfected with Fer-siRNA had a lower invasive ability, as compared with cells transfected with normal control siRNA. The experiments were repeated in triplicate. MMP-9 and MMP-2 expression in cells transfected with Fer-specific siRNA was detected by (C) reverse transcription-quantitative polymerase chain reaction and (D) western blotting. GAPDH was used as an internal control. Data are presented as the mean \pm standard deviation. ${ }^{*} \mathrm{P}<0.05$. Fer, feline sarcoma-related protein; MMP, matrix metalloproteinase; siRNA, small interfering RNA; NcT24, T24 cells transfected with normal control siRNA; SiT24, T24 cells transfected with Fer-specific siRNAs; ConT24, untreated T24 cells; OD, optical density.

in both the cytoplasm and nucleus of 55 (70.5\%) cancer tissues. Furthermore, it was observed that Fer protein expression significantly correlated with the tumor stage $(\mathrm{P}=0.042)$, histological grade $(\mathrm{P}=0.023)$ and lymph node status $(\mathrm{P}=0.014)$, but was not associated with age $(\mathrm{P}=0.459)$, gender $(\mathrm{P}=0.246)$ and tumor multiplicity $(\mathrm{P}=0.803)$ (Table II). The prognostic value of Fer for overall survival in bladder UCC patients was evaluated by comparing the patients with positive and negative Fer expression. According to the Kaplan-Meier survival analysis, bladder UCC patients with positive Fer expression had markedly lower overall survival rates than patients with negative Fer expression (log-rank value $=8.390 ; \mathrm{P}=0.0038 ;$ Fig. $1 \mathrm{E})$. These results suggest that the Fer expression status may be useful for predicting the overall survival of patients with bladder UCC.

Knockdown of the Fer gene using siRNA inhibits the migration of T24 cells. To determine the optimum cell model for investigating the role of Fer in bladder UCC, the mRNA and protein expression levels of Fer in various bladder UCC cells lines were evaluated. The protein and mRNA expression of Fer was upregulated in three bladder UCC cell lines (BIU-87, T24 and 5637), as compared with the normal bladder epithelium cell line, SV-HUC-1. Furthermore, high levels of Fer expression were observed in T24 cells compared with 5637 and BIU-87 cells (Fig. 2A and B). Therefore, T24 cells were selected to assess the effects of Fer silencing on bladder UCC cells by transfecting the cells with three positive Fer-siRNAs in order to obtain efficient and specific Fer depletion. As shown in Fig. 2C, the relative mRNA expression levels of Fer were significantly decreased by $72 \%$ in T24 cells transfected with siRNA1, as compared with the cells transfected with normal control siRNA $(\mathrm{P}=0.003)$, and were significantly lower than those cells transfected with siRNA2 and siRNA3. This result was also observed for the protein expresion levels (Fig. 2D). Therefore, Fer-siRNA1 was selected for the further analyses, as it demonstrated the most effective silencing effects on Fer in T24 cells. As shown in Fig. 2E and F, in monolayer wound healing assays, it was demonstrated that the cells transfected with Fer-siRNA showed a significantly reduced migration distance $(0.358 \pm 0.030 \mathrm{~mm})$, as compared with the cells transfected with the normal control siRNA group $(0.551 \pm 0.033 \mathrm{~mm})(\mathrm{P}<0.05)$.

Fer gene silencing inhibits cell invasion and suppresses MMP family protein expression in $T 24$ cells. To functionally confirm the role of Fer in aggressive bladder cancers, matrigel invasion assays were performed. The OD of the Fer-siRNA-transfected cells was significantly reduced compared with the OD of the T24 cells transfected with normal control siRNA $(0.635 \pm 0.066$ vs. $1.249 \pm 0.062$, respectively; $\mathrm{P}<0.05$; Fig. $3 \mathrm{~A}$ and $\mathrm{B}$ ). Previous studies have reported that MMPs are the principal mediators of 

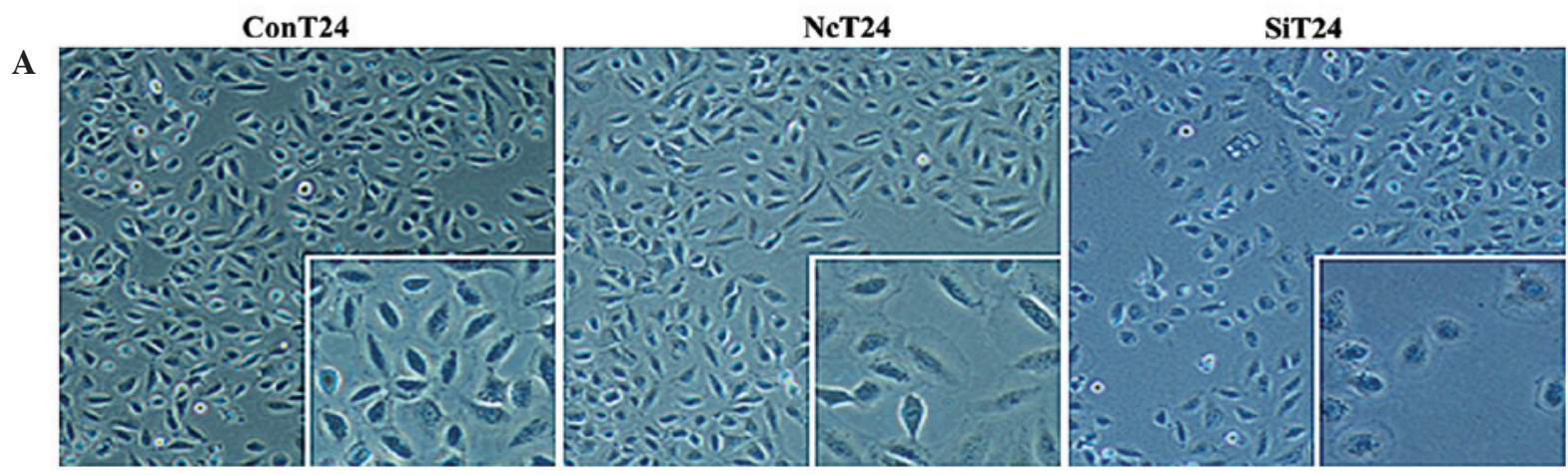

B

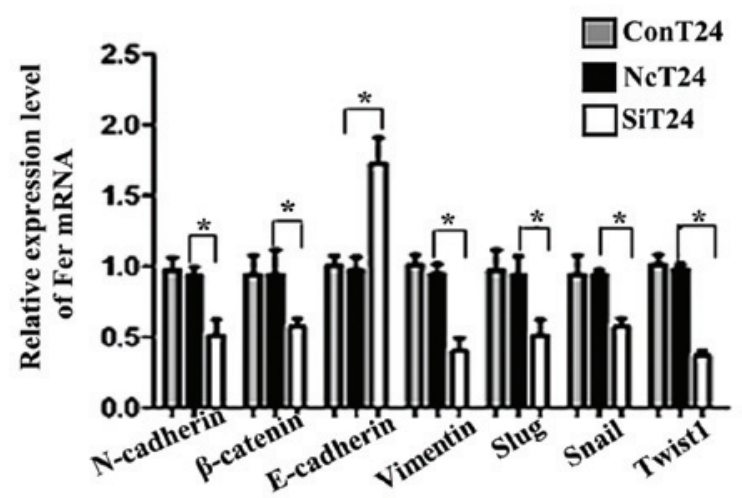

D

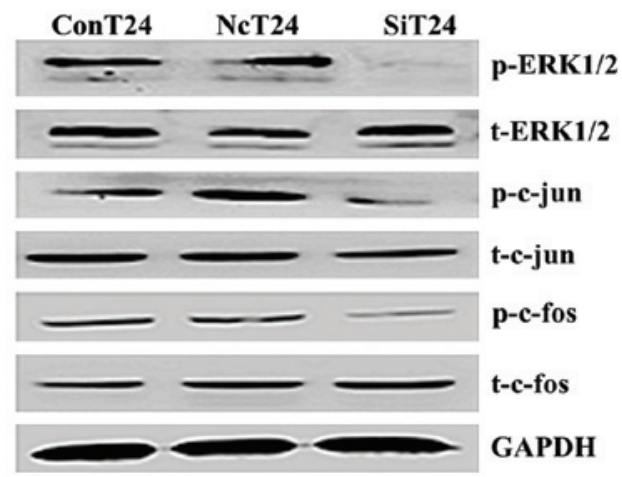

C

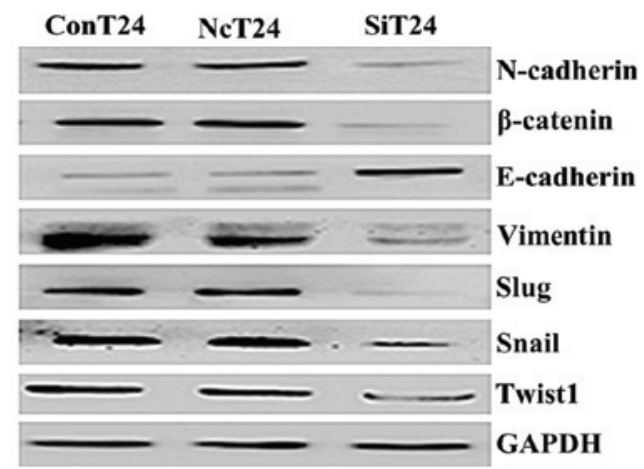

$\mathbf{E}$

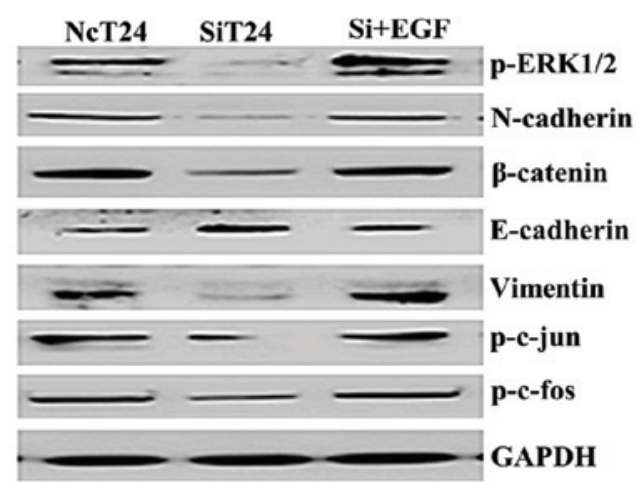

Figure 4. Knockdown of Fer induced a mesenchymal-epithelial transition-like phenotype and altered the expression of EMT-associated genes via the ERK/AP-1 pathway in T24 cells. (A) Light microscopy of T24 cells transfected with Fer-siRNA (magnification, x100; framed in white, x200). At 72 h following transfection, the majority of Fer-siRNA-transfected T24 cells were rounded, which is typical of an epithelial cobblestone appearance. Knockdown of Fer differentially regulated the (B) mRNA and (C) protein expression of EMT-associated proteins and transcription factors in T24 cells. GAPDH was used as an internal control. Data are presented as the mean \pm standard deviation. ${ }^{*} \mathrm{P}<0.05$. (D) The phosphorylation levels of ERK1/2 and AP-1 in T24 cells were decreased by silencing of Fer expression, as demonstrated in a western blot analysis. (E) T24 cells transfected with Fer-siRNA were treated with EGF ( 0.1 ng/ml) for 48 h, and the effect of EGF on the expression of EMT-associated proteins and the phosphorylation levels of ERK1/2 and AP-1 were detected by western blotting. Fer, feline sarcoma-related protein; EMT, epithelial-mesenchymal transition; ERK/AP-1, extracellular signal-regulated kinase/activator protein-1; siRNA, small interfering RNA; NcT24, T24 cells transfected with normal control siRNA; SiT24, T24 cells transfected with Fer-specific siRNAs; ConT24, untreated T24 cells; EGF, epidermal growth factor; Snail, snail family transcriptional repressor 1; Slug, snail family transcriptional repressor 2; Twist1, twist family bHLH transcription factor 1.

alterations in the cancer microenvironment during cancer metastasis (20). Therefore, the present study investigated whether the MMP protein family was involved in bladder UCC invasion and metastasis. As shown in Fig. 3C and D, the expression levels of MMP-2 and MMP-9 were significantly downregulated in Fer-siRNA-transfected T24 cells, as compared with the cells transfected with normal control siRNA $(\mathrm{P}<0.05)$.

Fer gene silencing induces changes in the morphology and markers of EMT in T24 cells via the ERK/AP-1 pathway. The morphology of T24 cells transfected with Fer-siRNA or normal control siRNA was analyzed by light microscopy. T24 cells transfected with normal control siRNA had an irregular fibroblastoid morphology, tight intercellular structure and a clear contour, while the majority of the Fer-siRNA-transfected T24 cells displayed a rounded shape, typical of an epithelial cobblestone appearance (Fig. 4A). These changes from a mesenchymal morphology to an epithelial morphology suggested that Fer silencing was able to reverse the EMT of T24 cells. As shown in Fig. 4B and C, silencing of Fer in T24 cells increased the expression of E-cadherin and decreased the expression of $\beta$-catenin, $\mathrm{N}$-cadherin and vimentin. In addition, the expression 
levels of the EMT-regulating transcription factors, Slug, Snail and Twist1, were downregulated in Fer-siRNA-transfected cells. Furthermore, there was a marked decrease in the expression of phospho-ERK1/2, p-c-jun and p-c-fos following Fer-knockdown in the T24 cells, but not total ERK/AP-1 (Fig. 4D). These findings suggest that inhibition of Fer by siRNA blocked the phosphorylation of ERK/AP-1. To further investigate whether the ERK/AP-1 pathway is involved in the Fer-induced EMT in T24 cells, an activator (EGF) of the ERK pathway was used. As shown in Fig. 4E, the EMT was promoted and the AP-1 levels were upregulated in siRNA-transfected cells incubated with EGF for $48 \mathrm{~h}$. These results suggest that the ERK/AP-1 pathway has an important role in Fer-induced EMT.

\section{Discussion}

It has been reported that Fer is extensively expressed in numerous mammalian cells, and is associated with tumor progression (21). However, the expression pattern and biological significance of Fer in bladder UCC are unclear. The present study demonstrated that Fer was significantly upregulated in bladder UCC tissues and cell lines, as compared with adjacent normal bladder tissues and the SV-HUC-1 normal human urothelial cell line, respectively. These findings indicate that Fer is involved in the progression of bladder UCC. In addition, an immunohistochemical analysis of bladder UCC specimens suggested that Fer expression was significantly associated with tumor stage, histological grade and lymph node status. Notably, Fer expression was an independent prognostic factor for a poor prognosis in patients with bladder UCC patients.

Several studies have reported that Fer is involved in tumor invasion and metastasis $(5,22,23)$; however, the role and cellular mechanisms of Fer in bladder UCC are not well-known. In the present study, siRNA was used to knockdown Fer expression in T24 cells, and the ability of the cells to migrate and invade was investigated. Notably, T24 cell migration and invasion was significantly reduced in cells transfected with Fer-siRNA. Increasingly it has been suggested that MMPs, which degrade the extracellular matrix and cell adhesion molecules, enhance cancer cell metastasis $(24,25)$. The present study demonstrated that knockdown of Fer expression was able to downregulate the expression of MMP-9 and MMP-2; thus suggesting that Fer may have an important role in the migration and invasion of T24 cells via the regulation of MMP gene expression.

During the process of metastasis, cancer cells often initiate the EMT, which is a dynamic cellular process thought to promote the acquisition of migratory and invasive abilities (26). During the EMT, morphological changes from the epithelial polarized morphology to the mesenchymal fibroblastoid morphology occur (27). Previous studies reported that Fer is involved in integrin/E-cadherin-mediated signaling pathways and has a role in regulating the cross-talk between cadherin-catenin complexes via focal adhesions (28-30). Furthermore, it was revealed that multiple small $\mathrm{G}$ protein/MAPK cascades were involved in the downstream signal transduction by Fps/Fes tyrosine kinases (31). The initiation of the EMT is dependent on the concomitant activity of MAPK pathways to induce the morphogenic process of the EMT (16).
In the present study, transfection of T24 cells with Fer-siRNA resulted in alterations in the morphology of T24 cells from a mesenchymal to an epithelial phenotype. In addition, silencing of Fer expression was shown to increase the expression of epithelial junction proteins (E-cadherin) and decrease the expression of mesenchymal markers (N-cadherin, vimentin and $\beta$-catenin). Furthermore, Fer-siRNA decreased the expression of Snail, Slug and Twist1 in T24 cells, which are known to potently modulate epithelial cell plasticity and induce the EMT phenotype $(12,32,33)$. Another significant finding was that Fer inhibition disrupted the ERK/AP-1 signaling pathway by suppressing the phosphorylation of ERK/AP-1. These results suggested that Fer silencing inhibited the EMT via suppression of the ERK/AP-1 signaling pathway.

In summary, the present study demonstrated that Fer was upregulated in patients with bladder UCC and that the Fer expression status was associated with tumor development and prognosis in these patients. In addition, it was shown that knockdown of Fer reduced the migration and invasion of T24 cells, and reversed the EMT by blocking the ERK/AP-1 signaling pathway. However, further studies are required to explore whether Fer-siRNA affected other upstream or downstream signaling molecules. These results suggested that targeting Fer signaling using novel approaches may be useful for reversing the EMT phenotype, which would likely result in the reversal of neoplasm recurrence and elimination of bladder UCC.

\section{Acknowledgements}

This study was supported by grants from the National Natural Science Foundation of China (grant nos. 81373005, 81072330 and 81202194) and by the Priority Academic Program Development of Jiangsu Higher Education Institutions.

\section{References}

1. Bo J, Yang G, Huo K, Jiang H, Zhang L, Liu D and Huang Y: microRNA-203 suppresses bladder cancer development by repressing bcl-w expression. FEBS J 278: 786-792, 2011.

2. Jemal A, Bray F, Center MM, Ferlay J, Ward E and Forman D: Global cancer statistics. CA Cancer J Clin 61: 69-90, 2011.

3. Ben-Dor I, Bern O, Tennenbaum T and Nir U: Cell cycle-dependent nuclear accumulation of the p94fer tyrosine kinase is regulated by its $\mathrm{NH} 2$ terminus and is affected by kinase domain integrity and ATP binding. Cell Growth Differ 10: 113-129, 1999.

4. Miyata Y,Kanda S, Sakai H and Greer PA: Feline sarcoma-related protein expression correlates with malignant aggressiveness and poor prognosis in renal cell carcinoma. Cancer Sci 104: 681-686, 2013.

5. Li H, Ren Z, Kang X, Zhang L, Li X, Wang Y, Xue T, Shen Y and Liu Y: Identification of tyrosine-phosphorylated proteins associated with metastasis and functional analysis of FER in human hepatocellular carcinoma cells. BMC Cancer 9: 366, 2009.

6. Zoubeidi A, Rocha J, Zouanat FZ, Hamel L, Scarlata E, Aprikian AG and Chevalier S: The Fer tyrosine kinase cooperates with interleukin-6 to activate signal transducer and activator of transcription 3 and promote human prostate cancer cell growth. Mol Cancer Res 7: 142-155, 2009.

7. Albeck JG and Brugge JS: Uncovering a tumor suppressor for triple-negative breast cancers. Cell 144: 638-640, 2011.

8. Allard P, Zoubeidi A, Nguyen LT, Tessier S, Tanguay S, Chevrette M, Aprikian A and Chevalier S: Links between Fer tyrosine kinase expression levels and prostate cell proliferation. Mol Cell Endocrinol 159: 63-77, 2000.

9. Stetler-Stevenson WG and Yu AE: Proteases in invasion: Matrix metalloproteinases. Semin Cancer Biol 11: 143-152, 2001. 
10. Thiery JP, Acloque H, Huang RY and Nieto MA: Epithelial-mesenchymal transitions in development and disease. Cell 139: 871-890, 2009.

11. Batlle E, Sancho E, Francí C, Domínguez D, Monfar M, Baulida J and García De Herreros A: The transcription factor snail is a repressor of E-cadherin gene expression in epithelial tumour cells. Nat Cell Biol 2: 84-99, 2000.

12. Bolós V, Peinado H, Pérez-Moreno MA, Fraga MF, Esteller M and Cano A: The transcription factor slug represses E-cadherin expression and induces epithelial to mesenchymal transitions: A comparison with Snail and E47 repressors. J Cell Sci 116: 499-511, 2003.

13. Singh A and Settleman J: EMT, cancer stem cells and drug resistance: An emerging axis of evil in the war on cancer. Oncogene 29: 4741-4751, 2010.

14. Sebolt-Leopold JS and Herrera R: Targeting the mitogen-activated protein kinase cascade to treat cancer. Nat Rev Cancer 4: 937-947, 2004.

15. Angel P and Karin M: The role of Jun, Fos and the AP-1 complex in cell-proliferation and transformation. Biochim Biophys Acta 1072: 129-157, 1991.

16. Raman M, Chen W and Cobb MH: Differential regulation and properties of MAPKs. Oncogene 26: 3100-3112, 2007.

17. Sobin DH and Wittekind C (eds): TNM Classification of Malignant Tumors. 6th edition. Wiley-Liss, New York, NY, 2003.

18. Miyamoto H, Miller JS, Fajardo DA, Lee TK, Netto GJ and Epstein JI: Non-invasive papillary urothelial neoplasms: The 2004 WHO/ISUP classification system. Pathol Int 60: 1-8, 2010.

19. Livak KJ and Schmittgen TD: Analysis of relative gene expression data using real-time quantitative PCR and the 2(-Delta Delta C(T)) Method. Methods 25: 402-408, 2001.

20. Groblewska M, Siewko M, Mroczko B and Szmitkowski M: The role of matrix metalloproteinases (MMPs) and their inhibitors (TIMPs) in the development of esophageal cancer. Folia Histochem Cytobiol 50: 12-19, 2012.

21. Hao QL, Heisterkamp N and Groffen J: Isolation and sequence analysis of a novel human tyrosine kinase gene. Mol Cell Biol 9: 1587-1593, 1989.

22. Orlovsky K, Theodor L, Malovani H, Chowers Y and Nir U: Gamma interferon down-regulates Fer and induces its association with inactive Stat3 in colon carcinoma cells. Oncogene 21: 4997-5001, 2002.
23. Ahn J, Truesdell P, Meens J, Kadish C, Yang X, Boag AH and Craig AW: Fer protein-tyrosine kinase promotes lung adenocarcinoma cell invasion and tumor metastasis. Mol Cancer Res 11: 952-963, 2013.

24. Deryugina EI and Quigley JP: Matrix metalloproteinases and tumor metastasis. Cancer Metastasis Rev 25: 9-34, 2006.

25. Shuman Moss LA, Jensen-Taubman S and Stetler-Stevenson WG: Matrix metalloproteinases: Changing roles in tumor progression and metastasis. Am J Pathol 181: 1895-1899, 2012.

26. Scheel C, Onder T, Karnoub A and Weinberg RA: Adaptation versus selection: The origins of metastatic behavior. Cancer Res 67: 11476-11479; discussion 11479-11480, 2007.

27. Yang $\mathbf{J}$ and Weinberg RA: Epithelial-mesenchymal transition At the crossroads of development and tumor metastasis. Dev Cell 14: 818-829, 2008

28. Craig AW and Greer PA: Fer kinase is required for sustained p38 kinase activation and maximal chemotaxis of activated mast cells. Mol Cell Biol 22: 6363-6374, 2002.

29. Kogata N, Masuda M, Kamioka Y, Yamagishi A, Endo A, Okada M and Mochizuki N: Identification of Fer tyrosine kinase localized on microtubules as a platelet endothelial cell adhesion molecule-1 phosphorylating kinase in vascular endothelial cells. Mol Biol Cell 14: 3553-3564,2003.

30. Piedra J, Miravet , Castaño J, Pálmer HG, Heisterkamp N, García de Herreros A and Duñach M: p120 Catenin-associated Fer and Fyn tyrosine kinases regulate beta-catenin Tyr-142 phosphorylation and beta-catenin-alpha-catenin interaction. Mol Cell Biol 23: 2287-2297, 2003.

31. Senis YA, Sangrar W, Zirngibl RA, Craig AW, Lee DH and Greer PA: Fps/Fes and Fer non-receptor protein-tyrosine kinases regulate collagen- and ADP-induced platelet aggregation. J Thromb Haemost 1: 1062-1070, 2003.

32. Cano A, Pérez-Moreno MA, Rodrigo I, Locascio A, Blanco MJ, del Barrio MG, Portillo F and Nieto MA: The transcription factor snail controls epithelial-mesenchymal transitions by repressing E-cadherin expression. Nat Cell Biol 2: 76-83, 2000 .

33. Liu AN, Zhu ZH, Chang SJ and Hang XS: Twist expression associated with the epithelial-mesenchymal transition in gastric cancer. Mol Cell Biochem 367: 195-203, 2012. 